\title{
Loss Calculation and Optimization Design of High Frequency Transformer
}

\author{
Lin Li", Keke Liu, Xiaoying Zhang, Ning Zhang \\ Department of Electrical and Electronics Engineering, North China Electric Power University, Beijing, China \\ Email address: \\ lilin@ncepu.edu.cn (Lin Li)
}

\section{To cite this article:}

Lin Li, Keke Liu, Xiaoying Zhang, Ning Zhang. Loss Calculation and Optimization Design of High Frequency Transformer. International Journal of Energy and Power Engineering. Special Issue: Numerical Analysis, Material Modeling and Validation for Magnetic Losses in Electromagnetic Devices. Vol. 5, No. 1-1, 2016, pp. 53-56. doi: 10.11648/j.ijepe.s.2016050101.18

\begin{abstract}
In this paper, the magnetization and loss properties are analyzed and compared of topical magnetic materials. The calculation methods are studied for the losses of the core and windings of the High Frequency Transformer (HFT). Based on the evaluation of the temperature increment, an optimization method is presented for the design of HFT. Finally, the losses of a test model of HFT is calculated and the results are compared with those tested.
\end{abstract}

Keywords: High Frequency Transformer, Magnetic Materials, Loss, Optimization

\section{Introduction}

In power electronic devices, such as DC/DC converter and solid state transformer, the operational frequencies of the transformers are chosen from several hundred $\mathrm{Hz}$ to several tens $\mathrm{kHz}$ in order to decrease their volumes and weights. This kind of transformers is called as High Frequency Transformer (HFT). The magnetization and loss mechanism of magnetic materials are related with the microscopic magnetization processes. In engineering application, the losses of soft magnetic material can usually be divided in three parts: eddy current loss, hysteresis loss and excess loss [1]. The Steinmetz formula is widely used to calculate the loss of magnetic core of transformer [2]. The losses of windings of high frequency transformer are related with the operation frequency, and the coefficient of alternative current resistance is used to reflect the skin effect and the proximity effect [3].

In this paper, the magnetization and loss properties are analyzed and compared of topical magnetic materials. The calculation methods of the losses of the core and windings of the HFT are analyzed. An optimization method is presented for the design of HFT. The losses of a test model of HFT is calculated and the results are compared with those tested.

\section{Magnetization and Loss Properties of Topical Magnetic Materials}

\subsection{Silicon Steel Sheets}

The superiorities of silicon steel sheets include: high saturation flux density, high stacking factor, high mechanical strength, good ductility, easy to cut, and low price, which let silicon steel sheets popularly used as the basic elements of lamination core of power transformer. But the eddy current and hysteresis losses of silicon steel sheets increase quickly with the increase of operation frequency, even if the sheets are ultrathin strips.

\subsection{Amorphous Alloys}

Amorphous alloys are also called as metallic glasses. When amorphous alloys are cooled from the liquid state and solidified as noncrystalline materials whose cooling rate is estimated to be in the range $10^{5}-10^{6} \mathrm{~K} / \mathrm{sec}$. The ribbons of amorphous alloys are usually a few millimeters wide, 25-35 $\mathrm{nm}$ thick, and meters to kilometers in length. The losses of amorphous alloys for unit volume are much lower than silicon steel sheets, especially in high frequencies. Therefore, amorphous alloys are usually chosen as the magnetic material for high frequency small capacity transformers and distribution power transformers. Maximum saturation magnetization is in the range $1.5-1.9 \mathrm{~T}$, which is much larger 
than ferrites. Based on the design principle of transformer, rising the working flux density of the transformer core can decrease the turn number of the windings, and further the volume and weight of the high frequency transformer.

\subsection{Nanocrystalline Alloys}

A related class of nanocrystalline alloys is made by adding small amounts of $\mathrm{Cu}$ and $\mathrm{Nb}$ to an $\mathrm{Fe}-\mathrm{Si}-\mathrm{B}$ amorphous alloy. The most-studied composition is Fe74Si15B7Cu1Nb3. The $\mathrm{Cu}$ is believed to enhance nucleation of crystallites and the $\mathrm{Nb}$ to inhibit their growth. The saturation flux density of nanocrystalline alloys is high as amorphous alloys, and the high frequencies losses of nanocrystalline alloys for unit volume are lower than amorphous alloys. The price for unit mass of nanocrystalline alloys is so much higher than amorphous alloys that the material has not been applied in industry. In other hand, because the material is extremely brittle, it is difficult to manufacture magnetic core of nanocrystalline alloys with gaps.

\subsection{Soft Ferrites}

Ferrites have good properties of high frequencies losses. As a soft magnetic material, ferrites are widely used in small high frequency switching power supply, in which the operation frequency is from several $\mathrm{kHz}$ to several $\mathrm{MHz}$. The saturation flux density of ferrites is lower as $0.2 \mathrm{~T}$ so that the operation frequency chosen must be higher to make up the deficiency and lower the turn number of the windings of the transformer. Because ferrites are farinose, and unfavorable machining, the cores of ferrites are annular or closed UU shape without gaps.

\subsection{Optimized Magnetic Material for High Frequency Transformer}

The loss properties of silicon steel sheets, amorphous alloys, nanocrystalline alloys, and soft ferrites in $10 \mathrm{kHz}$ and $50 \mathrm{kHz}$ are shown in Fig.1. Loss properties and saturation flux densities of four magnetic core of silicon steel sheets, amorphous alloys, nanocrystalline alloys, and soft ferrites, are tested and listed in table 1 . The saturation flux densities, losses for unit volume, machining properiesy and prices for unit mass are the main factors to choose magnetic material for high frequency transformers. From Fig. 1 and table 1, it can be seen that the optimized magnetic materials are amorphous alloys or nanocrystalline alloys for high frequency transformer with several $\mathrm{kHz}$ operation frequency.

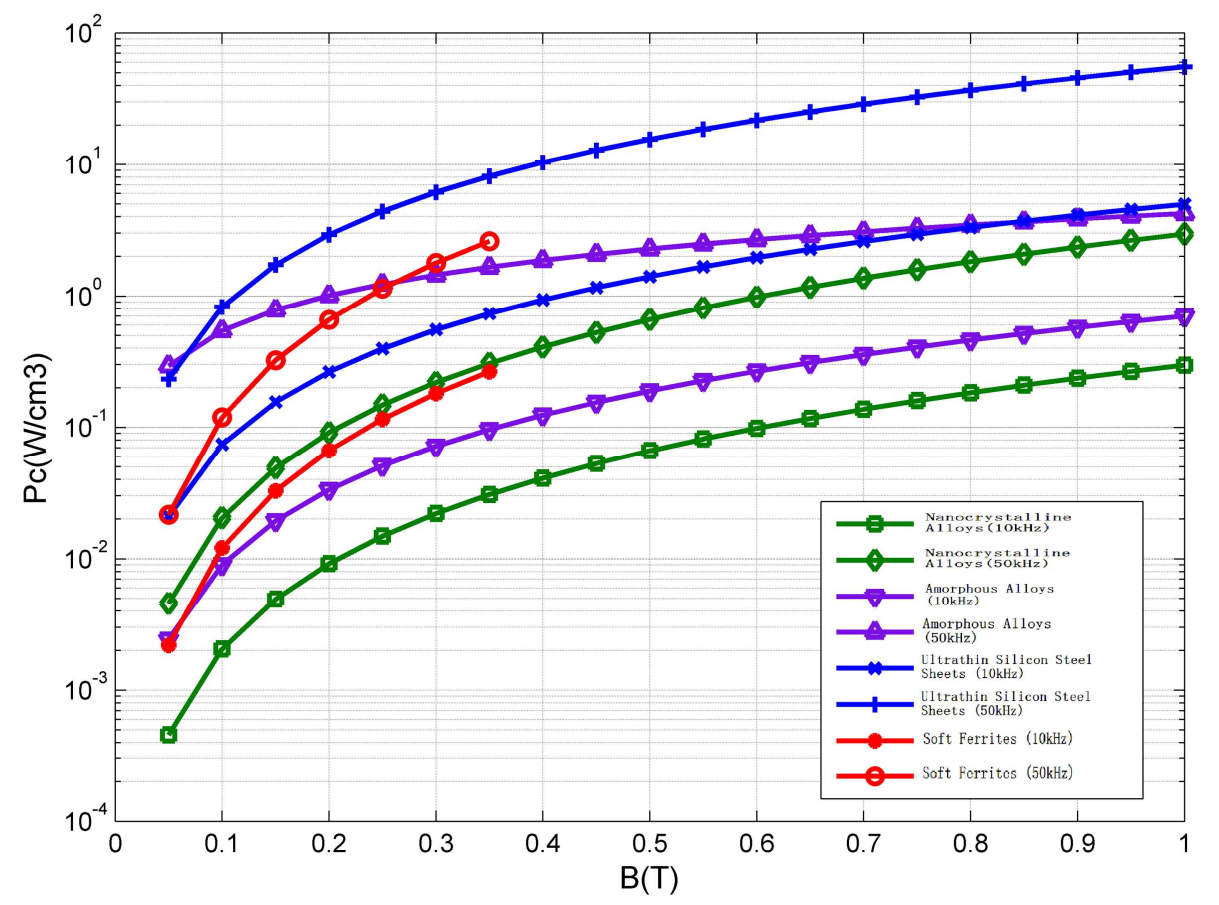

Figure 1. Loss properties of ultrathin silicon steel sheets, amorphous alloys, nanocrystalline alloys, and soft ferrites.

Table 1. Loss properties and saturation flux densities of four magnetic cores.

\begin{tabular}{lll}
\hline $\begin{array}{l}\text { Magnetic } \\
\text { material }\end{array}$ & $\begin{array}{l}\text { Saturation flux } \\
\text { densities (T) }\end{array}$ & Loss properties $([\mathbf{W} / \mathbf{c m} 3)$ \\
\hline $\begin{array}{l}\text { Ferrites } \\
\text { amorphous alloys }\end{array}$ & $0.35 \mathrm{~T}$ & $P_{c}=0.1334 B^{2.464}[\mathrm{~T}] f^{1.422}[\mathrm{kHz}]$ \\
$\begin{array}{l}\text { nanocrystalline } \\
\text { alloys }\end{array}$ & $1.2 \mathrm{~T}$ & $P_{c}=0.0536 B^{1.891}[\mathrm{~T}] f^{1.117}[\mathrm{kHz}]$ \\
$\begin{array}{l}\text { ultrathin silicon } \\
\text { steel sheets }\end{array}$ & $1.5 \mathrm{~T}$ & $P_{c}=0.0111 B^{2.161}[\mathrm{~T}] f^{1.428}[\mathrm{kHz}]$ \\
\hline
\end{tabular}

\section{Losses of High Frequency Transformer}

\subsection{Loss of Magnetic Core}

Usually, losses of magnetic core can be divided in three parts: eddy current loss, hysteresis loss and excess loss. The losses of magnetic core per unit mass can be calculated by [1]:

$$
P_{c}=C_{h} B_{m}^{\alpha} f+C_{e} B_{m}^{2} f^{2}+C_{e x} B_{m}^{1.5} f^{1.5}
$$


where $C_{\mathrm{h}}, C_{\mathrm{e}}$, and $C_{\mathrm{ex}}$ are the coefficients of eddy current loss, hysteresis loss and excess loss, respectively. $f$ is the operation frequency of high frequency transformer, and $B_{\mathrm{m}}$ is the maximum magnetic flux density of the core. $\alpha$ is an undetermined index, which is related with specified magnetic material. Another reduced core loss calculation formula is as follows [2]

$$
P_{c}=C_{w} k B_{m}^{\alpha} f^{\beta}
$$

where $C_{\mathrm{w}}$ is the coefficients related with the waveform of the magnetic flux density, 1 for sinusoidal wave, $\pi / 4$ for square wave, and $2 / 3$ for triangle wave. $k, \alpha$ and $\beta$ are undetermined indexes related with specified magnetic material. (2) is also called Steinmetz formula.

\subsection{Losses of the Windings}

The loss of winding of transformer can be calculated by:

$$
P_{w}=k_{r} R_{D C} I_{r m s}^{2}
$$

where $R_{\mathrm{DC}}$ is the direct current resistance of the winding, and $I_{\mathrm{rms}}$ is the root mean square current. $k \mathrm{r}\left(=R_{\mathrm{AC}} / R_{\mathrm{DC}}\right)$ is the coefficient of alternative current resistance, which can be expressed as follows [3]:

$$
\begin{aligned}
& k_{r}(X, m)=X\left[\frac{\sinh (2 X)+\sin (2 X)}{\cosh (2 X)-\cos (2 X)}\right. \\
& \left.+\frac{2\left(m^{2}-1\right)}{3} \frac{\sinh (X)-\sin (X)}{\cosh (X)+\cos (X)}\right]
\end{aligned}
$$

where $\mathrm{m}$ is the number of layers of the winding, and $X=\frac{h_{c}}{0.071 \sqrt{f}}$, in which $h_{\mathrm{c}}$ is the thickness of the conductor wires.

\subsection{A Model of High Frequency Transformer}

A model of high frequency transformer is made and shown in Fig.2, whose basic parameters are listed in table 2. The zero-load losses of the transformer for different frequencies are tested that can be used to represented the losses of core. The calculation formula of loss of core is obtained by means of the tested data, and it is as follows:

$$
P_{c}=0.00354 B_{m}^{1.75} f^{1.527}
$$

Table 2. Basic parameters of the model of high frequency transformer.

\begin{tabular}{lllll}
\hline $\begin{array}{l}\text { Magnetic } \\
\text { material }\end{array}$ & $\begin{array}{l}\text { Saturation flux } \\
\text { densities (T) }\end{array}$ & $\begin{array}{l}\text { Voltages of } \\
\text { windings (V) }\end{array}$ & $\begin{array}{l}\text { Frequenc } \\
\text { y (Hz) }\end{array}$ & $\begin{array}{l}\text { Capacity } \\
\text { (kVA) }\end{array}$ \\
\hline $\begin{array}{l}\text { 0.3mm silicon } \\
\text { steel sheets }\end{array}$ & 0.7 & $560 / 160$ & 400 & 1.5 \\
\hline
\end{tabular}

The calculated and test losses of core are listed in table 3. The calculated and test losses of windings are listed in table 4 . It can be seen from table 3-4 that the errors are small between the calculated and the tested losses of the core and windings.

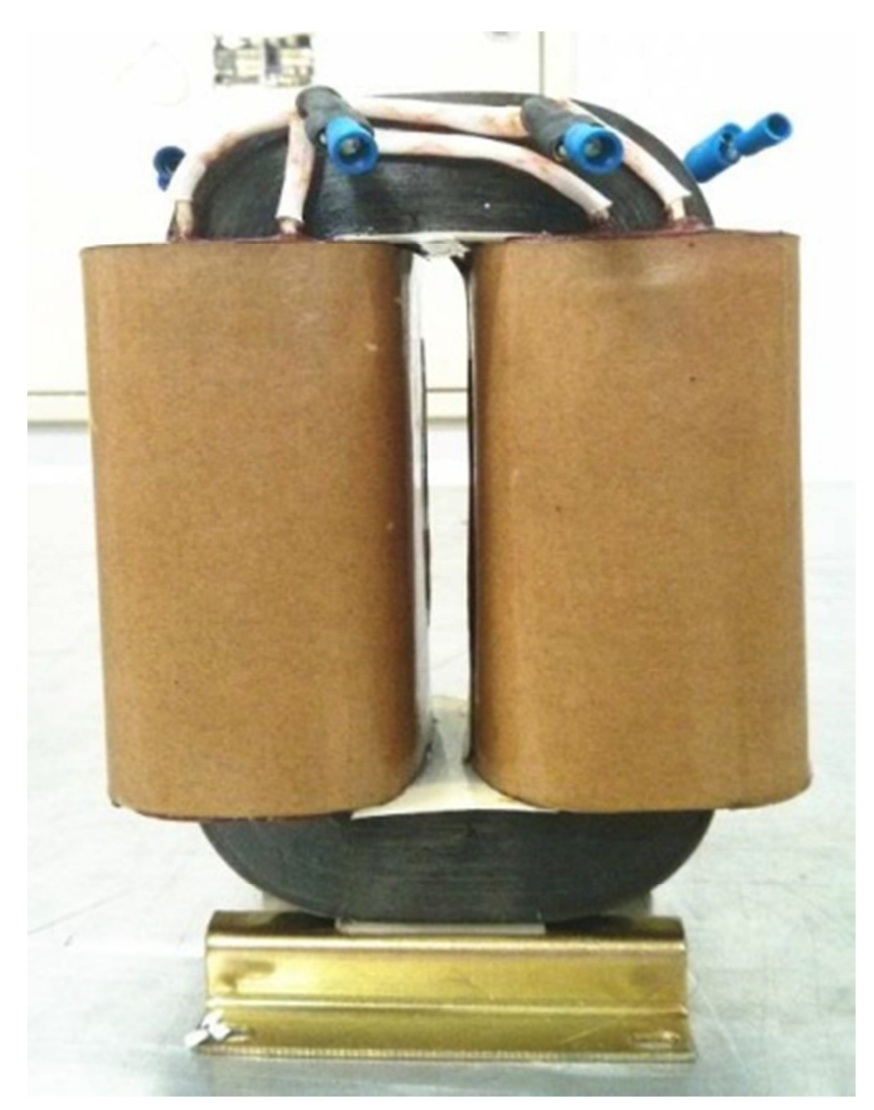

Figure 2. Model of high frequency transformer.

Table 3. Calculated and test losses of core.

\begin{tabular}{llll}
\hline $\boldsymbol{f}(\mathbf{H z})$ & Calculated $\boldsymbol{P c}(\mathbf{W})$ & Tested $\boldsymbol{P c}(\mathbf{W})$ & Error(\%) \\
\hline 50 & 0.740 & 0.7 & 5.71 \\
60 & 0.935 & 0.9 & 3.89 \\
100 & 1.900 & 2.0 & -5.00 \\
200 & 5.304 & 5.5 & -3.56 \\
300 & 10.470 & 10.35 & 1.15 \\
\hline
\end{tabular}

Table 4. Calculated and test losses of windings.

\begin{tabular}{llll}
\hline $\boldsymbol{f}(\mathbf{H z})$ & Calculated $\boldsymbol{P w}(\mathbf{W})$ & Tested $\boldsymbol{P w}(\mathbf{W})$ & Error(\%) \\
\hline 50 & 32.5 & 31.05 & 4.67 \\
60 & 32.5 & 31.05 & 4.67 \\
100 & 32.8 & 31.25 & 4.96 \\
200 & 33.3 & 32.68 & 1.90 \\
300 & 35.3 & 35.10 & 0.57 \\
400 & 38.2 & 38.13 & 0.18 \\
\hline
\end{tabular}

\section{Optimization Design of High Frequency Transformer}

One of the optimization design objects of high frequency transformer is to determine the optimum magnetic flux density $B_{\text {opt }}$ corresponding to the minimum total loss $P_{\mathrm{t}}$ of the transformer and the determined operation frequency. Let the derivative of the total loss $P_{\mathrm{t}}$ to the magnetic flux density $B$ equal to zero, that is,

$$
\frac{\mathrm{d} P_{\mathrm{t}}}{\mathrm{d} B}=\frac{\mathrm{d} P_{c}}{d B}+\frac{d P_{w}}{d B}=0
$$


We can obtained the optimum magnetic flux density $B_{\text {opt }}$ as:

$$
\begin{aligned}
& \mathrm{B}_{\text {opt }}^{\boldsymbol{\beta}+1}=\left[\boldsymbol{K}_{1} \boldsymbol{U}_{\text {in }} \cdot 10^{4} \cdot \boldsymbol{M L T} \boldsymbol{T} \boldsymbol{\rho}_{1} \boldsymbol{I}_{1}^{2}+\right. \\
& \left.\boldsymbol{K}_{2} \boldsymbol{U}_{\text {out }} \cdot 10^{4} \cdot \boldsymbol{M L T} \cdot \boldsymbol{\rho} \boldsymbol{I}_{2}^{2}\right] / 4.44 \boldsymbol{A}_{c} \boldsymbol{f}^{\boldsymbol{\alpha}+1} \boldsymbol{k} \boldsymbol{\beta} \boldsymbol{V}_{\boldsymbol{c}}
\end{aligned}
$$

where $k, \alpha$ and $\beta$ are the indexes related with specified magnetic material and the same as that in (2). $U_{\text {in }}$ and $U_{\text {out }}$ are the voltages of the primary winding and the secondary winding, respectively. $I_{1}$ and $I_{2}$ are the currents of the primary winding and the secondary winding, respectively. $V_{c}$ and $A_{c}$ are the volume and across area of the magnetic core, respectively. $K_{1}$ and $K_{2}$ are the coefficient of alternative current resistance of the primary winding and the secondary winding, respectively. $M L T$ is the average turn length of the windings and $\rho$ is the resistivity of conductor of of the windings.

For one kind of amorphous alloys, the calculation formula of the loss of magnetic core is: $P_{c}\left(\mathrm{~W} / \mathrm{cm}^{3}\right)=0.0306 B^{1.74} f(k H z)^{1.51}$. The optimization design results of a $10 \mathrm{kVA}$ high frequency transformer are shown in table 5. For one kind of nanocrystalline alloys, the calculation formula of the loss of magnetic core is: $P_{c}\left(\mathrm{~W} / \mathrm{cm}^{3}\right)=0.008 B^{1.982} f\left(\mathrm{kHz}^{1.621}\right.$. The optimization design results of a $10 \mathrm{kVA}$ high frequency transformer are shown in table 6 .

\section{Conclusions}

Based on the study of the paper, we obtained the following conclusions:

(1) For the large capacity high frequency, the optimization magnetic materials are amorphous alloys and nanocrystalline alloys which have better magnetization and loss properties.

(2) The Steinmetz formula can be used to calculate the loss of core, and the method of coefficient of alternative current resistance to the losses of windings of HFT.

\begin{tabular}{|c|c|c|c|c|c|c|}
\hline $\mathbf{f}(\mathrm{kHz})$ & Вор(T) & $\mathrm{Vc}(\mathrm{cm} 3)$ & S/Ve & $\operatorname{Pc}(W)$ & $\operatorname{Pcu}(\mathbf{W})$ & $\operatorname{Pt}(W)$ \\
\hline 10 & 0.2939 & 259.9911 & 39.84205 & 30.5711 & 53.5436 & 84.1146 \\
\hline 20 & 0.1574 & 241.6788 & 41.37723 & 27.3037 & 47.7399 & 75.0436 \\
\hline 30 & 0.1106 & 229.5788 & 43.55803 & 25.8983 & 45.3952 & 71.2935 \\
\hline 40 & 0.0951 & 203.5538 & 49.12706 & 26.3105 & 46.1546 & 72.4651 \\
\hline 50 & 0.077 & 192.6796 & 51.89963 & 27.059 & 44.5896 & 71.6486 \\
\hline 80 & 0.0472 & 232.6637 & 42.98049 & 26.1927 & 45.7791 & 71.9718 \\
\hline 100 & 0.0375 & 250.1911 & 39.96945 & 26.4792 & 46.2359 & 73.705 \\
\hline
\end{tabular}

Table 5. Optimization design results of a $10 \mathrm{kVA}$ amorphous alloys high frequency transformer.

Table 6. Optimization design results of a $10 \mathrm{kVA}$ nanocrystalline alloys high frequency transformer.

\begin{tabular}{lllllll}
\hline $\mathbf{f}(\mathbf{k H z})$ & $\mathbf{B o p}(\mathbf{T})$ & $\mathbf{V c}(\mathbf{c m} 3)$ & $\mathbf{S} / \mathbf{V c}$ & $\mathbf{P c}(\mathbf{W})$ & $\mathbf{P c u}(\mathbf{W})$ & $\mathbf{P t}(\mathbf{W})$ \\
\hline 10 & 0.5633 & 129 & 77.51938 & 13.8365 & 27.6104 & 41.4469 \\
20 & 0.3217 & 113.9947 & 87.7233 & 12.3767 & 24.6451 & 37.0218 \\
30 & 0.2349 & 105.8522 & 94.4713 & 11.8885 & 23.5591 & 35.4476 \\
50 & 0.1486 & 104.5664 & 95.6330 & 10.8533 & 21.8397 & 32.693 \\
80 & 0.1011 & 104.5664 & 95.6330 & 10.8344 & 21.4794 & 32.3138 \\
100 & 0.0799 & 112.2372 & 89.0970 & 10.4679 & 20.9212 & 31.3891 \\
\hline
\end{tabular}

[3] P.L. Dowell. Effect of eddy currents in transformer windings[J]. Proc. Inst. Elect. Eng., 113, pp1387-1394, 1996.

\section{References}

[1] Boglietti A, Cavagnino A, Lazzari M, et al. Predicting iron losses in soft magnetic materials with arbitrary voltage supply: an engineering approach [J]. IEEE Trans. Magn., 39(2): pp.981-989, March 2003.

[2] Bertotti G. General properties of power losses in soft ferromagnetic materials[J]. IEEE Trans. Magn., 24(1): pp621-630, January, 1988.

[4] I. D. Mayergoyz, G. Friedman, and C. Salling, "Comparison of the classical and generalized Preisach hysteresis models with experiments," IEEE Trans. Magn., vol. 25, no. 5, pp. 39253927, Sep. 1989.

[5] E. Barbisio, F. Fiorillo, and C. Ragusa, "Predicting loss in magnetic steels under arbitrary induction waveform and with minor hysteresis loops," IEEE Trans. Magn., vol. 40, no. 4, pp. 1810-1819, Jul. 2004. 